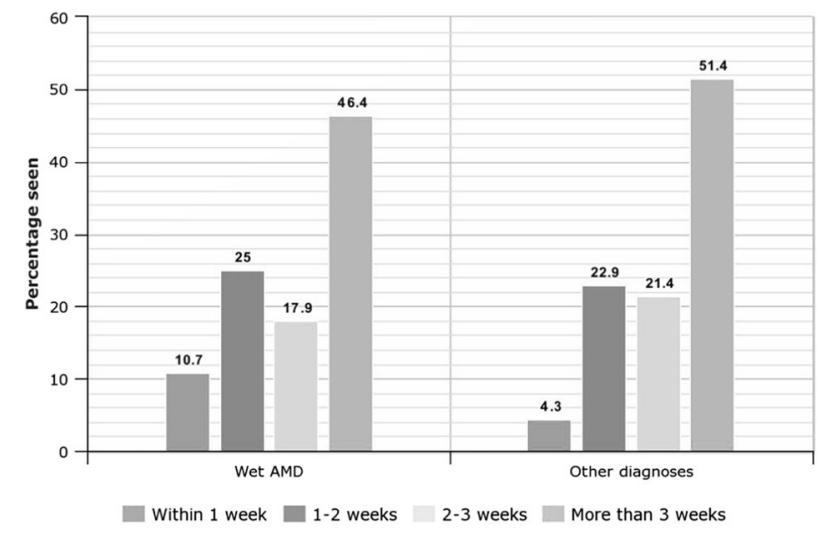

Fig. 2 Delay between referral and specialist review by diagnosis

Acknowledgements We thank the Thames Valley Macular Group Rapid Access Guidelines developed by SM Downes, C Moorman, SL Watson and L Jenkins in collaboration with the Oxfordshire LOC.

\section{Compliance with ethical standards}

Conflict of interest Susan Downes Disclosure: PI on relevant commercially sponsored trials in last 5 years: Novartis, Bayer, and
Alcon. Prof Susan Downes has received honoraria in the past 15 years from Novartis and Bayer for speaking at educational meetings, as well as travel expenses from Ely Lilly as chair for diabetic retinopathy screening meetings, and Novartis to attend educational meetings in Medical Retina pre 2010. She is a PI on a number of commercial trials (Novartis, Roche, Bayer, Allergen, among others), and has received a bursary for a research nurse practitioner, and equipment (visudyne pump for photodynamic therapy) and funding to carry out genetic testing for a research project from Novartis. She also has has been a co-PI or Pl on grants from Wellcome, UK Fighting Blindness RP, Fight for Sight and UK NIHR/CRN funding. The remaining authors declare that they have no conflict of interest.

\section{References}

1. The Royal College of Ophthalmologists. Age-Related Macular Degeneration: Guidelines for Management 2013. https://www. rcophth.ac.uk/wp-content/uploads/2014/12/2013-SCI-318RCOphth-AMD-Guidelines-Sept-2013-FINAL-2.pdf

2. The Royal College of Ophthalmologists. Wet AMD Rapid Access Referral Form 2010. https://www.rcophth.ac.uk/wp-content/uploa ds/2015/04/2010-SCI-048-AMD-Electronic-Referral-Form-edited. pdf

\title{
Accuracy of pinhole visual acuity at an urban Indian hospital
}

\author{
Rajesh S. Kumar ${ }^{1,2} \cdot$ Mahalakshmi V. Rackenchath ${ }^{1} \cdot$ A. V. Sathidevi ${ }^{1} \cdot$ Sriharsha Nagaraj $^{1} \cdot$ Caitlin A. Moe $^{3}$. \\ Robert L. Stamper ${ }^{4}$ Jeremy D. Keenan $\mathbb{1 D}^{3,4}$
}

Received: 16 August 2018 / Accepted: 7 September 2018 / Published online: 19 October 2018

(c) The Royal College of Ophthalmologists 2018

The World Health Organization-endorsed rapid assessment of avoidable blindness (RAAB) survey employs pinhole acuity to distinguish between refractive error versus conditions not correctable with eyeglasses, but few studies have validated this approach [1].

Jeremy D. Keenan

jeremy.keenan@ucsf.edu

1 Narayana Nethralaya Eye Hospital, Bangalore, India

2 Cleveland Clinic Abu Dhabi, Abu Dhabi, UAE

3 Francis I Proctor Foundation, University of California, San Francisco, CA, USA

4 Department of Ophthalmology, University of California, San Francisco, CA, USA

\section{Methods}

Ethical committees at the University of California, San Francisco and Narayana Nethralya Eye Hospital approved this study. A consecutive series of patients aged $\geq 50$ years visiting the refraction clinic at Narayana Nethralaya Eye Hospital (Bangalore, India) in September 2015 had presenting vision and pinhole vision assessed using an ETDRS chart in a fully illuminated room, and then had a manifest refraction by an experienced optometrist. Analyses are reported with bootstrapped $95 \%$ confidence intervals with resampling at the participant level to account for nonindependence of eyes. 


\section{Results}

We assessed 204 eyes from 104 individuals (mean age $63.4 \pm 7.8$ years, $51.9 \%$ female). Mean spherical equivalent after manifest refraction was -0.31 (95\% confidence interval $[\mathrm{CI}]:-0.07$ to -0.60$)$. Mean uncorrected visual acuity was $0.43 \log$ MAR units (95\% CI: 0.38-0.49), mean best corrected visual acuity (BCVA) was 0.16 (95\% CI: $0.13-$ 0.19 ) and mean pinhole acuity was 0.15 (95\% CI: 0.12 0.18). Pinhole acuity had high agreement with BCVA (intraclass correlation coefficient $0.97,95 \%$ CI: 0.96-0.98; Fig. 1). On average, pinhole acuity was less than a letter different from BCVA (mean 0.4 letters better, $95 \%$ limits of agreement by Bland-Altman method: four letters worse to five letters better). The magnitude of improvement on pinhole testing was correlated with the magnitude of spherical equivalent from refraction (Spearman's rho $=0.68, P<$ 0.001; Fig. 2).

Of 204 eyes, 21 (10.3\%) had visual impairment even after subjective refraction (BCVA worse than 20/60). When treated as a diagnostic test for visual impairment not correctable with eyeglasses, pinhole acuity provided high discriminative ability, with an area under the receiver operating characteristics (ROC) curve of 0.99 (95\% CI: 0.97-1.0). Pinhole acuity worse than $20 / 60$ was $85.7 \%$ sensitive (95\% CI: $59.1-100 \%)$ and $100 \%$ specific $(98.0-100 \%)$ for detecting visual impairment not correctable with glasses, and had a positive predictive value of $100 \%$ (95\% CI: $81.4-100 \%)$.

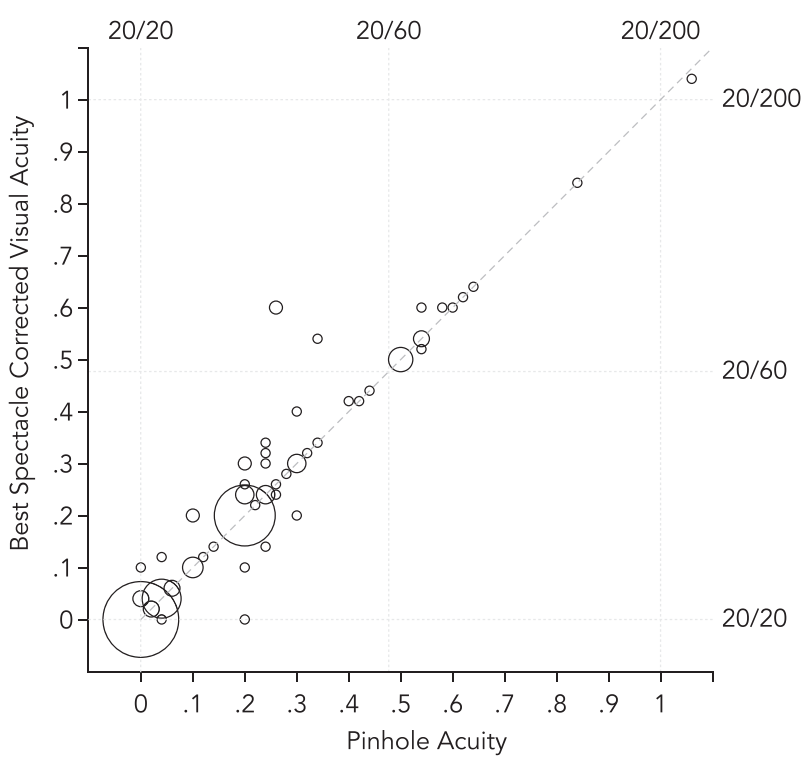

Fig. 1 Correlation between measurements of best spectacle corrected visual acuity and pinhole acuity. Points are weighted; the area of the circles represents the number of observations at each coordinate

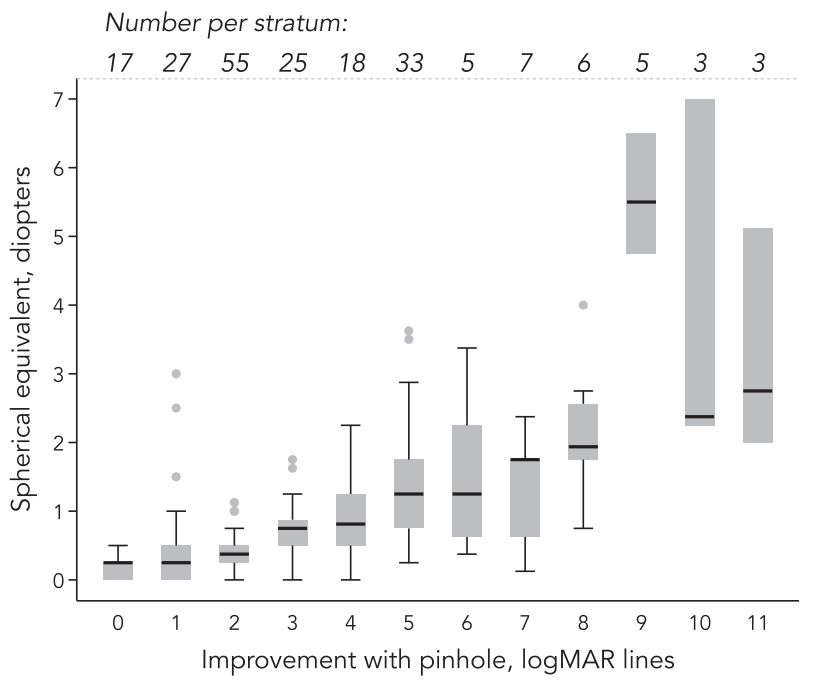

Fig. 2 Relationship between improvement with pinhole occlusion and refractive error. Eyes were stratified according to how many lines of improvement were achieved with pinhole occlusion. The distribution of spherical equivalent for each stratum is depicted as a box-andwhiskers plot

\section{Conclusions}

Previous studies of patients with diabetic retinopathy or low vision found that pinhole acuity was biased relative to BCVA, with pinhole acuity $\sim 1$ line worse than BCVA $[2,3]$. In contrast, we found essentially no bias in this population from a refraction clinic in India. The reason for the discrepancy is unclear, but the pinhole occluder may cause more visual degradation in those with retinal pathology than in a general population like ours [2]. The estimates of sensitivity and specificity of pinhole acuity as a test for visual impairment are consistent with prior reports, and suggest that estimates of refractive error based on pinhole occlusion should not overestimate the prevalence of disease [4].

In summary, pinhole acuity agreed well with BCVA and was a specific test for visual impairment not correctable with eyeglasses. These results suggest that pinhole occlusion is a valid gauge of refractive error in the RAAB survey or other community-based surveys.

Funding This work was funded by the Fortisure Foundation, That Man May See, and Research to Prevent Blindness.

\section{Compliance with ethical standards}

Conflict of interest The authors declare that they have no conflict of interest. 


\section{References}

1. Kuper H, Polack S, Limburg H. Rapid assessment of avoidable blindness. Community Eye Health. 2006;19:68-9.

2. Eagan SM, Jacobs RJ, Demers-Turco PL. Study of luminance effects on pinhole test results for visually impaired patients. Optom Vis Sci. 1999;76:50-8.
3. Sun JK, et al. Visual acuity testing using autorefraction or pinhole occluder compared with a manual protocol refraction in individuals with diabetes. Ophthalmology. 2011;118:537-42. https://doi.org/ 10.1016/j.ophtha.2010.07.022

4. Loewenstein JI, Palmberg PF, Connett JE, Wentworth DN. Effectiveness of a pinhole method for visual acuity screening. Arch Ophthalmol. 1985;103:222-3.

\title{
Time to drop the phenylephrine from the paediatric cycloplegia protocol: informing practice through audit
}

\author{
Sue Llewellyn ${ }^{1,2} \cdot$ Payal Khandelwal $^{2,3} \cdot$ Selina Glaze ${ }^{2,3} \cdot$ Peter Thomas $^{2,4,5} \cdot$ Annegret Dahlmann-Noor $\mathbb{D}^{2,4,5}$
}

Received: 25 September 2018 / Accepted: 4 October 2018 / Published online: 23 October 2018

(c) The Royal College of Ophthalmologists 2018

Cycloplegic retinoscopy is the gold standard refraction approach in children. Various protocols are in use to achieve cycloplegia during outpatient appointments. Many use not only the muscarinergic antagonist, cyclopentolate (CP), which paralyses the ciliary muscle, but also the alphaadrenergic agonist, phenylephrine (PE), which as a mydriatic paralyses the iris constrictor, but has no effect on the ciliary muscle. Whilst mydriasis facilitates visualisation of the retinoscopy reflex, there is a risk of underestimating hypermetropia. Published evidence suggests that repeated

This work was presented at the annual meeting of the British Isles Paediatric Ophthalmology and Strabismus Association, Manchester, October 3-5, 2018.

\section{Annegret Dahlmann-Noor}

annegret.dahlmann-noor@nhs.net

Bedford Hospital Trust, Kempston Road, Bedford, UK

2 Moorfields at Bedford Hospital, Children's Eye Clinic, Enhanced Services Centre, Kimbolton Road, Bedford, UK

3 Cambridgeshire Community Services NHS Trust, Unit 3, Meadow Lane, St Ives PE27 4LG, UK

4 Paediatric Service, Moorfields Eye Hospital, 162 City Road, London EC1V 2PD, UK

5 NIHR Biomedical Research Centre at Moorfields Eye Hospital and UCL Institute of Ophthalmology, 162 City Road, London EC1V 2PD, UK instillation of $\mathrm{CP}$ only is effective even for brown and very dark irides [1-3].

To develop a Patient Group Directive (PGD) we carried out a two-cycle audit (CA18/PA/02). The first round evaluated our current protocol: blue iris, CP $1 \%$ once $(0.5 \%$ if age $<3$ months); brown iris, CP/PE $2.5 \%$ once; very dark iris, CP/PE twice, $10-15$ min apart; repeated if pupils still constrict on pentorch illumination. As standard, we set full dilation in $90 \%$ within $30 \mathrm{~min}$, i.e. the level expected for blue irides with a single $\mathrm{CP}$ drop [4].

In the second round, we only included children with brown or very dark iris, administering $\mathrm{CP}$ twice or three times, respectively, $10-15$ min apart.

Table 1 Ethnic background and iris colour of children assessed in this audit

\begin{tabular}{llllll}
\hline & \multicolumn{2}{l}{ Round 1 } & & \multicolumn{2}{l}{ Round 2 } \\
\cline { 6 - 6 } & $n$ & $\%$ & & $n$ & $\%$ \\
\hline Ethnicity & & & & \\
Afro-Caribbean & 5 & 9 & 10 & 20 \\
Asian & 7 & 13 & 19 & 37 \\
Caucasian & 44 & 79 & 20 & 39 \\
Chinese & & & 1 & 2 \\
Other & & & 1 & 2 \\
Iris colour & 32 & 57 & & \\
Blue & 11 & 20 & 14 & 27 \\
Brown & 13 & 23 & 37 & 73 \\
Very dark & & & & \\
\hline
\end{tabular}

